\title{
Phase behavior of an amphiphilic molecule in the presence of two solvents by dissipative particle dynamics
}

\author{
Ching-I Huang*, Yu-Jeng Chiou, Yi-Kang Lan \\ Institute of Polymer Science and Engineering, National Taiwan University, No. 1, Roosevelt Road, Sec. 4, Taipei 106, Taiwan
}

Received 15 June 2006; received in revised form 20 October 2006; accepted 10 December 2006

Available online 8 January 2007

\begin{abstract}
We examine the phase behavior of $\mathrm{A}_{m} \mathrm{~B}_{n}$ amphiphilic molecules in the presence of two solvents $\mathrm{X}_{2}$ and $\mathrm{Y}_{2}$, which are strongly selective for $\mathrm{A}$ and $\mathrm{B}$, respectively, by dissipative particle dynamics (DPD). We find that increasing the immiscibility parameter between the two solvents not only drives a macrophase separation into two phases $\mathrm{X}_{2}$-rich and $\mathrm{Y}_{2}$-rich for systems at less concentrated regimes, but also expands the ordered microphase region at more concentrated regimes. It even induces a sequential transition of various ordered structures. This is not surprising since increasing the solvent immiscibility parameter enhances the preferentiality of $\mathrm{X}_{2}$ for $\mathrm{A}$ and $\mathrm{Y}_{2}$ for $\mathrm{B}$, and thus qualitatively varies the degree of molecular asymmetry in the amphiphilic molecules. In general, our current results reveal that the DPD simulation method has successfully captured the phase separation behavior of an amphiphilic molecule in the presence of two solvents. However, we find that the packing order of the spherical micelles is greatly affected by the finite size of the simulation box. As such, it becomes difficult to examine the most stable packing array of spheres via the DPD method. Still, DPD reveals a possible spherical order of A15, which has been observed in some amphiphilic molecule systems.
\end{abstract}

(C) 2007 Elsevier Ltd. All rights reserved.

Keywords: Dissipative particle dynamics; Amphiphilic; Solvent immiscibility

\section{Introduction}

Amphiphilic molecules, such as surfactants, lipids, and diblock copolymers, continue to attract a lot of attention due to the fact that they can self-assemble into a rich variety of morphologies [1-7]. Many of the amphiphilic molecule systems with valuable technological applications contain amphiphilic molecules, oil, and water [7]. Due to the fact that water and oil are strongly immiscible, the swelling ability of both hydrophilic and hydrophobic groups in the amphiphilic molecules varies with the solvent ratio and solvent amount. As a result, various microstructures with different interfacial curvatures, such as lamellae, normal (oil-in-water) and reverse (waterin-oil) bicontinuous phase, normal and reverse cylinders, and normal and reverse spheres, form in the ternary mixtures of

\footnotetext{
* Corresponding author. Tel.: +886 2 33665886; fax: +886 233665237 .

E-mail address: chingih@ntu.edu.tw (C.-I. Huang).
}

amphiphilic molecules, water, and oil [2,7]. Indeed, phase behavior of an amphiphilic molecule in two solvents becomes very complicated as it involves the effects of the immiscibility between two solvents, solvent amount, solvent ratio, and the selectivity of two solvents for each amphiphilic group. Most of the theoretical research has focused on the examination of phase behavior and the interfacial properties of an amphiphilic molecule in the presence of two very immiscible solvents [813]. To our knowledge, however, the effects of solvent miscibility degree on the resulting microstructure formation of amphiphilic molecules have not been fully examined. In this paper, we employ dissipative particle dynamics (DPD) to simulate the phase behavior of an amphiphilic molecule in the presence of two solvents. In particular, the ternary phase diagrams at various degrees of the solvent immiscibility parameter are constructed.

DPD is a coarse-grained mesoscale simulation technique [14], which has recently been successfully applied to study the 
mesophase behavior of a variety of amphiphilic molecule systems [12,13,15-22]. Groot and Madden first applied DPD to examine the microphase separation behavior of linear $\mathrm{A}_{m} \mathrm{~B}_{n}$ diblock copolymer melts [16]. By varying the A composition and the effective $\mathrm{A} / \mathrm{B}$ segregation parameter, various ordered structures, such as lamellae (L), gyroid (G), perforated lamellae (PL), hexagonally packed cylinders (C), and spheres (S), have been obtained via DPD. In further, the phase diagram constructed by DPD is in a near quantitative agreement with that predicted by self-consistent mean-field theory [23]. In the amphiphilic molecule solutions, the related current DPD studies were mainly focused on the less concentrated regimes. For example, Cao et al. employed DPD to simulate the aggregation behavior of poly(ethylene oxide)-poly(propylene oxide) block copolymers in aqueous solutions [22]. In particular, the effects of the copolymer architecture and concentration on the formed micelle type and size were examined. Their results are in a qualitatively good agreement with experiments [24]. Rekvig et al. have demonstrated that the DPD simulations can successfully describe the partitioning behavior of the surfactant along the interface between water and oil [12]. Schulz et al. applied DPD to simulate the self-assembling behavior of surfactant $\mathrm{C}_{10} \mathrm{E}_{4}$ in the presence of water and oil [13]. Their simulated structure results by varying the water/ oil ratio are in good agreement with the experimental phase diagram [25]. Though these solution studies demonstrated that the DPD simulation method is an appropriate technique to examine the phase behavior of amphiphilic molecules in the presence of two solvents, their results neither extend to the concentrated regimes nor consider the effects of solvent miscibility degree.

In this paper, we thus aim to simulate the phase behavior of an amphiphilic molecule in the presence of two solvents by DPD simulation method. In particular, the effects of the immiscibility parameter between two solvents, the solvent ratio, and the volume fraction of amphiphilic molecules, are analyzed. For simplicity, we consider a symmetric molecule and a asymmetric amphiphilic molecule, which are represented by two beads $\left(A_{1} B_{1}\right)$ and four beads $\left(A_{1} B_{3}\right)$, respectively. Two solvents $\mathrm{X}_{2}$ and $\mathrm{Y}_{2}$ are strongly selective for $\mathrm{A}$ and $\mathrm{B}$, respectively. Later, we will demonstrate that the effects of adding two strongly selective solvents on the resulting phase behavior of $\mathrm{A}_{1} \mathrm{~B}_{1}$ and $\mathrm{A}_{1} \mathrm{~B}_{3}$ are qualitatively consistent. Hence, similar results should also hold true qualitatively in other amphiphilic molecules $\left(\mathrm{A}_{m} \mathrm{~B}_{n}\right)$ which initially form different ordered phases, such as G, PL, and S. Our DPD results provide a more complete understanding of the rich and complex phase behavior that amphiphilic molecules exhibit when two solvents are added.

\section{DPD simulation method}

In the DPD simulation, the time evolution of motion for a set of interacting particles is solved by Newton's equation. For simplicity, we assume that the masses of all particles are equal to 1 . The force acting on the $i$ th particle $\overrightarrow{f_{i}}$ contains three parts: a conservative force $\vec{F}_{i j}^{\mathrm{C}}$, a dissipative force $\vec{F}_{i j}^{\mathrm{D}}$, and a random force $\vec{F}_{i j}^{\mathrm{R}}$, i.e.,

$\overrightarrow{f_{i}}=\sum_{i \neq j}\left(\vec{F}_{i j}^{\mathrm{C}}+\vec{F}_{i j}^{\mathrm{D}}+\vec{F}_{i j}^{\mathrm{R}}\right)$

where the sum is over all other particles within a certain cutoff radius $r_{\mathrm{c}}$. As this short-range cut-off counts only local interactions, $r_{\mathrm{c}}$ is usually set to 1 so that all lengths are measured relative to the particle radius.

The conservative force $\vec{F}_{i j}$ is a soft repulsive force and given by

$\vec{F}_{i j}^{\mathrm{C}}=\left\{\begin{array}{cc}a_{i j}\left(1-\frac{r_{i j}}{r_{\mathrm{c}}}\right) \overrightarrow{n_{i j}} & r_{i j}<r_{\mathrm{c}} \\ 0 & r_{i j} \geq r_{\mathrm{c}}\end{array}\right.$

where $a_{i j}$ is the repulsive interaction parameter between particles $i$ and $j, \overrightarrow{r_{i j}}=\overrightarrow{r_{i}}-\overrightarrow{r_{j}}, r_{i j}=\left|\overrightarrow{r_{i j}}\right|$, and $\overrightarrow{n_{i j}}=\overrightarrow{r_{i j}} / r_{i j}$. The repulsion parameter $a_{i j}$ is often related to the FloryHuggins interaction parameter $\chi_{i j}$ by the following equation [15]

$a_{i j}(T)=a_{i i}+3.497 k_{\mathrm{B}} T \chi_{i j}(T)$ for $\rho=3$

$a_{i j}(T)=a_{i i}+1.451 k_{\mathrm{B}} T \chi_{i j}(T)$ for $\rho=5$

where $\rho$ is the particle density of the system. The term $a_{i i}$, which corresponds to the repulsion parameter between particles of the same type $i$, is determined by matching the water compressibility as [15]

$a_{i i}=75 k_{\mathrm{B}} T / \rho$

The dissipative force $\vec{F}_{i j}^{\mathrm{D}}$ is a hydrodynamic drag force and given by

$\vec{F}_{i j}^{\mathrm{D}}=\left\{\begin{array}{cc}-\gamma \omega^{\mathrm{D}}\left(r_{i j}\right)\left(\overrightarrow{n_{i j}} \cdot \overrightarrow{v_{i j}}\right) \overrightarrow{n_{i j}} & r_{i j}<r_{\mathrm{c}} \\ 0 & r_{i j} \geq r_{\mathrm{c}}\end{array}\right.$

where $\gamma$ is a friction parameter, $\omega^{\mathrm{D}}$ is a $r$-dependent weight function vanishing for $r \geq r_{\mathrm{c}}$, and $\overrightarrow{v_{i j}}=\overrightarrow{v_{i}}-\overrightarrow{v_{j}}$.

The random force $\vec{F}_{i j}^{\mathrm{R}}$ corresponds to the thermal noise and has the form of

$\vec{F}_{i j}^{\mathrm{R}}=\left\{\begin{array}{cc}\sigma \omega^{\mathrm{R}}\left(r_{i j}\right) \theta_{i j} \vec{n}_{i j} & r_{i j}<r_{\mathrm{c}} \\ 0 & r_{i j} \geq r_{\mathrm{c}}\end{array}\right.$

where $\sigma$ is a parameter, $\omega^{\mathrm{R}}$ is also a weight function, $\theta_{i j}(t)$ is a randomly fluctuating variable. Note that these two forces $\vec{F}_{i j}^{\mathrm{D}}$ and $\vec{F}_{i j}^{\mathrm{R}}$ also act along the line of centers and conserve linear and angular momentums. There is an independent random function for each pair of particles. Also there is a relation between both constants $\gamma$ and $\sigma$, which is as follows [15]

$\sigma^{2}=2 \gamma k_{\mathrm{B}} T$

In our simulations, $\gamma=4.5$ and the temperature $k_{\mathrm{B}} T=1$. As such, $\sigma=3.0$ according to Eq. (7).

In order for the steady-state solution to the equation of motion to be the Gibbs ensemble and for the fluctuationdissipation theorem to be satisfied, it has been shown [26] 
that only one of the two weight functions $\omega^{\mathrm{D}}$ and $\omega^{\mathrm{R}}$ can be chosen arbitrarily,

$\omega^{\mathrm{D}}(r)=\left[\omega^{\mathrm{R}}(r)\right]^{2}$

which, in further, is usually taken as

$\omega^{\mathrm{D}}(r)=\left[\omega^{\mathrm{R}}(r)\right]^{2}=\left\{\begin{array}{cc}\left(r_{\mathrm{c}}-r_{i j}\right)^{2} & r_{i j}<r_{\mathrm{c}} \\ 0 & r_{i j} \geq r_{\mathrm{c}}\end{array}\right.$

Finally, the spring force $\vec{f}_{i} \mathrm{~S}$, which acts between the connected beads in a molecule, has the form of

$\vec{f}_{i}^{\mathrm{S}}=\sum_{j} C \overrightarrow{r_{i j}}$

where $C$ is a harmonic type spring constant for the connecting pairs of beads in a molecule, and is chosen to be equal to 4 (in terms of $k_{\mathrm{B}} T$ ) [15].

Note that a modified version of the velocity-Verlet algorithm is used here to solve the Newtonian equation of motion [27]

$r_{i}(t+\Delta t)=r_{i}(t)+v_{i}(t) \cdot \Delta t+\frac{1}{2} f_{i}(t) \cdot \Delta t^{2}$

$\tilde{v}_{i}(t+\Delta t)=v_{i}(t)+\lambda f_{i}(t) \cdot \Delta t$

$f_{i}(t+\Delta t)=f_{i}\left[r_{i}(t+\Delta t)+\tilde{v}_{i}(t+\Delta t)\right]$

$v_{i}(t+\Delta t)=v_{i}(t)+\frac{1}{2} \Delta t \cdot\left[f_{i}(t)+f_{i}(t+\Delta t)\right]$

In particular, herein, we choose $\lambda=0.65$ and $\Delta t=0.05$.

\section{System}

In simulating the phase separation behavior of $\mathrm{A}_{m} \mathrm{~B}_{n}$ amphiphilic molecules in the presence of two solvents $X_{2}$ and $\mathrm{Y}_{2}$ by DPD, we assume that each component has the same volume per segment (bead). We choose two types of molecules, one is symmetric $\mathrm{A}_{1} \mathrm{~B}_{1}$ and the other is asymmetric $A_{1} B_{3}$. The solvents $X_{2}$ and $Y_{2}$ are strongly selective for $A$ and $\mathrm{B}$, respectively, i.e., $\mathrm{X}_{2}\left(\mathrm{Y}_{2}\right)$ likes $\mathrm{A}(\mathrm{B})$ instead of $\mathrm{B}(\mathrm{A})$. In particular, we set $a_{\mathrm{AY}}=a_{\mathrm{BX}}=100.54>a_{\mathrm{AX}}=a_{\mathrm{BY}}=25$. When the particle density $\rho=3$, the dimensionless repulsion parameter (i.e., in terms of $k_{\mathrm{B}} T$ ) between equal particles $a_{\mathrm{II}}$ in Eq. (4) is set equal to 25 to resemble the Flory interaction parameter $\chi_{\mathrm{II}}=0, \mathrm{I}=\mathrm{A}, \mathrm{B}, \mathrm{X}_{2}, \mathrm{Y}_{2}$. Parameter $a_{\mathrm{AB}}$ is also set at 100.54, which corresponds to $\chi_{\mathrm{AB}}=21.6$ according to Eq. (3). Therefore, the effective A/B interaction parameter $\left(\chi_{\mathrm{AB}} N\right)_{\mathrm{eff}}$ calculated by the following equation $[16,19]$

$\left(\chi_{\mathrm{AB}} N\right)_{\mathrm{eff}}=\frac{\chi_{\mathrm{AB}} N}{1+3.9 N^{-0.51}}$

for $A_{1} B_{1}$ and $A_{1} B_{3}$ is equal to 11.55 and 29.56, respectively. According to the prediction by self-consistent mean-field theory [23], these two amphiphilic molecules form the stable $\mathrm{L}$ phase and the hexagonally packed cylinders of $\mathrm{A}\left(\mathrm{C}_{\mathrm{A}}^{\mathrm{HEX}}\right)$, respectively. In order to simulate the effects of the immiscibility parameter between the two solvents, we choose $a_{\mathrm{XY}}=25,30$, and 40 , which corresponds to $\chi_{\mathrm{XY}}=0,1.43$ and 4.29 , respectively. As such, the systems with lower values of the volume fraction of amphiphilic molecules $\bar{\phi}_{\mathrm{C}}$ may remain in the disordered solution state at $a_{\mathrm{XY}}=0$, and undergo a macrophase separation into $\mathrm{X}_{2}$-rich and $\mathrm{Y}_{2}$-rich phases when $a_{\mathrm{XY}}$ increases to 30 and 40 . In addition, we adopt a 3D lattice with at least $10 \times 10 \times 10$ grids to assure that the side length of our simulation box is significantly larger than the radius of gyration of these amphiphilic molecules. In each pattern, the red, green, blue, and purple colors correspond to component A, B, X, and $\mathrm{Y}$, respectively.

\section{Results and discussion}

Fig. 1 illustrates the morphology variation of $A_{1} B_{1}$ with the addition of solvent $X_{2}$. When existing alone, $A_{1} B_{1}$ forms the stable L phase, as expected. As solvent $\mathrm{X}_{2}$ is added, because of the attraction of $X_{2}$ toward $A$, solvent $X_{2}$ moves into the A-rich layers. As a result, the systems with the surfactant volume fraction $\bar{\phi}_{\mathrm{C}} \geq 0.7$ form a lamellar $\mathrm{A} / \mathrm{X}_{2}$-rich and B-rich segregated structures. With increasing amount of $X_{2}$, because of the increasing degree of swelling by $\mathrm{X}_{2}$, we observe that the A-rich layers become thicker, and these amphiphilic molecules form a mutually parallel bilayer structure in the presence of $\mathrm{X}_{2}\left(0.6 \geq \bar{\phi}_{\mathrm{C}} \geq 0.2\right)$. With continuously adding $\mathrm{X}_{2}$ such that $\bar{\phi}_{\mathrm{C}} \leq 0.1$, relatively small amounts of $\mathrm{A}_{1} \mathrm{~B}_{1}$ evolve from the bilayer structure into a rod-like micelle with $\mathrm{A}$ facing outward and B facing inward. Note that due to the significant immiscibility between $X_{2}$ and $B$, the amount of $X_{2}$ which can be dissolved in the A-rich layers is expected to have a maximum, and therefore the extra addition of $\mathrm{X}_{2}$ may form a phase of its own. That is, the bilayer structure in the solvent $X_{2}$ formed at $0.2 \leq \bar{\phi}_{\mathrm{C}} \leq 0.6$ may reveal the possibility of two-macrophase separation into $1 \mathrm{~L}$ phase and 1 disordered (D) $\mathrm{X}_{2}$-rich phase. In order to further identify this issue, we expand our DPD simulation box from $10 \times 10 \times 10$ to $15 \times 15 \times 15$, and present the resulting pattern for $\bar{\phi}_{\mathrm{C}}=0.5$ in Fig. 1 . It is clear that the thickness of the $\mathrm{X}_{2}$-rich layers simulated in a box of $15 \times 15 \times 15$ is around 6.15 grids, which is significantly larger than 4.0 in $10 \times 10 \times 10$. Similar results have also been observed in other systems at $0.2 \leq \bar{\phi}_{\mathrm{C}} \leq 0.6$, indicating that these systems actually form a two-phase coexistence of $1 \mathrm{~L}$ and $1 \mathrm{D}$. As solvents $X_{2}$ and $Y_{2}$ have opposite selectivity for $A$ and $B$, the morphology variation of $A_{1} B_{1}$ with the addition of solvent $Y_{2}$ is similar to that of $A_{1} B_{1}+X_{2}$. In general, our current DPD results for a symmetric amphiphilic molecule in the presence of a strongly selective solvent are consistent with those for a symmetric $\mathrm{AB}$ block copolymer in the presence of a homopolymer A based on the self-consistent mean-field theory [28]. Note that when the solvent selectivity is slight, no macrophase separation occurs as $\bar{\phi}_{\mathrm{C}}$ decreases. The addition of an A-selective solvent $\mathrm{X}_{2}$ thus acts in a manner that corresponds qualitatively to increasing the $\mathrm{A}$ 
(a)

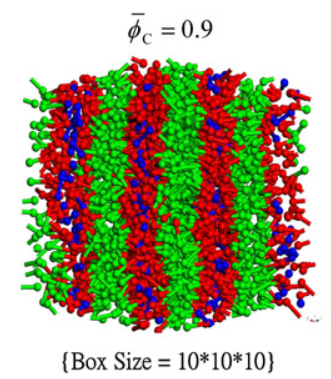

(b)

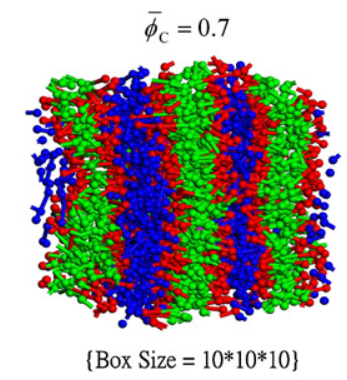

(c)
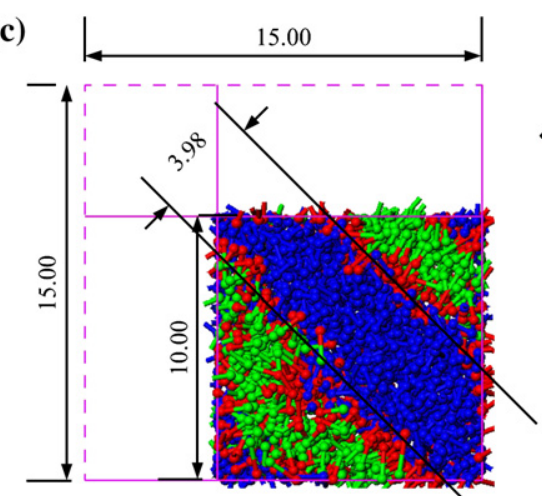

$\{$ Box Size $=10 * 10 * 10\}$

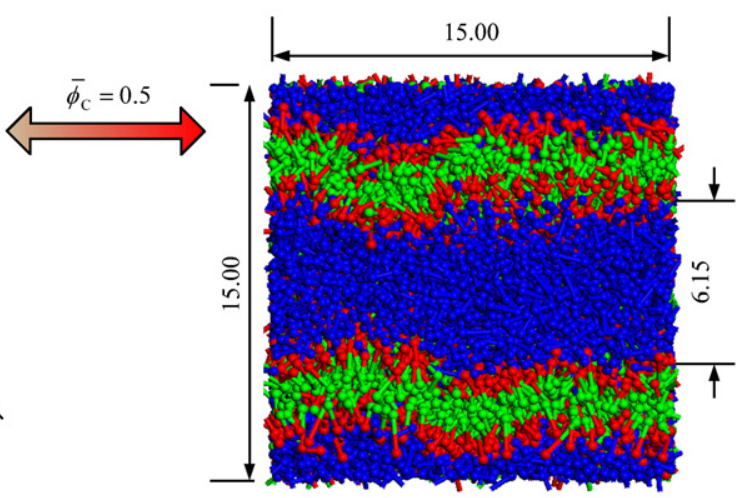

$\{$ Box Size $=15 * 15 * 15\}$

(d)

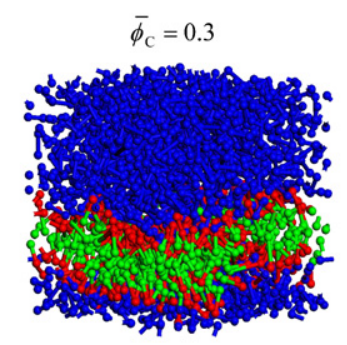

$\{$ Box Size $=10 * 10 * 10\}$ (e)

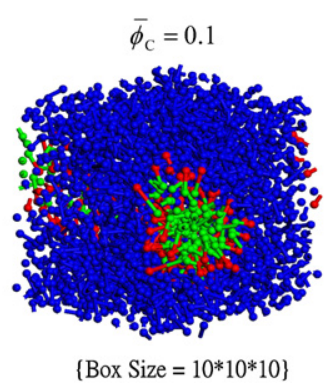

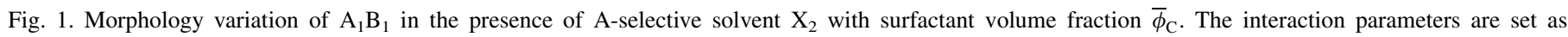

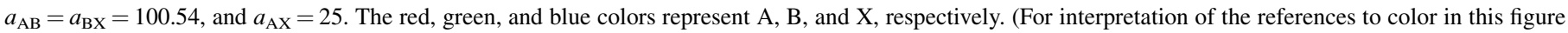
legend, the reader is referred to the web version of this article.)

composition $f$. In this case, one may expect that a sequence of $\mathrm{L} \rightarrow \mathrm{C}_{\mathrm{B}}^{\mathrm{HEX}} \rightarrow \mathrm{B}$-formed spheres $\rightarrow \mathrm{D}$ occurs, as frequently observed in the diblock copolymers in the presence of a selective solvent [29].

Fig. 2 presents a series of phase diagrams for $A_{1} B_{1}$ in the presence of solvents $X_{2}$ and $Y_{2}$, which are obtained by DPD simulations and shown in $\mathrm{AB}-\mathrm{X}_{2}-\mathrm{Y}_{2}$ composition triangles, at various values of the immiscibility parameter between two solvents $a_{\mathrm{XY}}$. The Ith corner in the triangle represents a system composed of $100 \%$ component I. When these two solvents are miscible, i.e., $a_{\mathrm{XY}}=25$, as shown in Fig. 2(a), we observe that for the solutions with $\bar{\phi}_{\mathrm{C}} \geq 0.7$, no matter what the volume fraction ratio of $\mathrm{X}_{2}$ and $\mathrm{Y}_{2}\left(\bar{\phi}_{\mathrm{X}_{2}} / \bar{\phi}_{\mathrm{Y}_{2}}\right)$ is, they will evolve into a lamellar structure. The typical pattern is presented in Fig. 3(a), where $\bar{\phi}_{\mathrm{C}}=0.7, \bar{\phi}_{\mathrm{X}_{2}} / \bar{\phi}_{\mathrm{Y}_{2}}=4 / 6$, and $a_{\mathrm{XY}}=25$. This is due to the fact that solvents $\mathrm{X}_{2}$ and $\mathrm{Y}_{2}$ have a very strong affinity to $\mathrm{A}$ and $\mathrm{B}$, respectively. Therefore, even if $X_{2}$ and $Y_{2}$ are miscible, a small amount of solvents $X_{2}$ and $\mathrm{Y}_{2}$ still accumulates into the A-rich and B-rich layers, respectively. However, when the solutions are in the intermediate concentration $\left(\bar{\phi}_{\mathrm{C}}=0.2-0.6\right)$, as the amount of these two solvents $X_{2}$ and $Y_{2}$ exceeds the maximum value which can be dissolved, respectively, in the A-rich and B-rich domains, they undergo a macrophase separation into a lamellar $\mathrm{A} / \mathrm{X}_{2}$-rich and $\mathrm{B} / \mathrm{Y}_{2}$-rich phase and a disordered $\mathrm{X}_{2} / \mathrm{Y}_{2}$ phase, as shown in Fig. 3(b), where $\bar{\phi}_{\mathrm{C}}=0.4, \bar{\phi}_{\mathrm{X}_{2}} / \bar{\phi}_{\mathrm{Y}_{2}}=4 / 6$, and $a_{\mathrm{XY}}=25$. Once the volume fraction of $\mathrm{A}_{1} \mathrm{~B}_{1}$ becomes very low, such as $\bar{\phi}_{\mathrm{C}} \leq 0.1$, the molecules no longer form the lamellar structure and instead a micelle-like structure with $\mathrm{B}$ facing outward and A facing inward (when $\mathrm{Y}_{2}$ is more than $\mathrm{X}_{2}$ ) or with $\mathrm{B}$ facing inward and $\mathrm{A}$ facing outward (when $\mathrm{X}_{2}$ is more than $\mathrm{Y}_{2}$ ) is formed.

As $a_{\mathrm{XY}}$ increases to 30 , the significant immiscibility parameter between two solvents not only drives a macrophase separation into $\mathrm{X}_{2}$-rich and $\mathrm{Y}_{2}$-rich phases for systems at lower concentration values of $\bar{\phi}_{\mathrm{C}}$, but also enlarges the 1 ordered $\mathrm{L}$ $\left(\mathrm{A} / \mathrm{X}_{2}\right.$-rich and $\mathrm{B} / \mathrm{Y}_{2}$-rich) microphase region till the middle of the phase triangle, as seen in the corresponding phase 
(a)

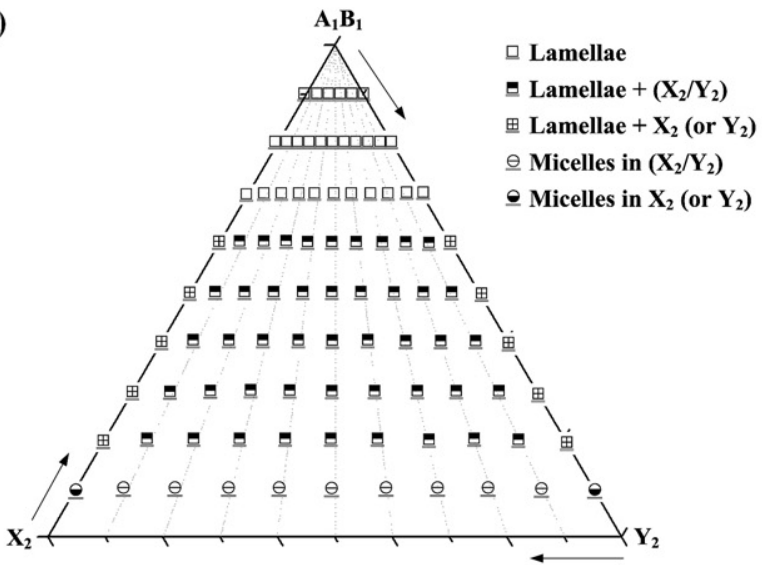

(b)

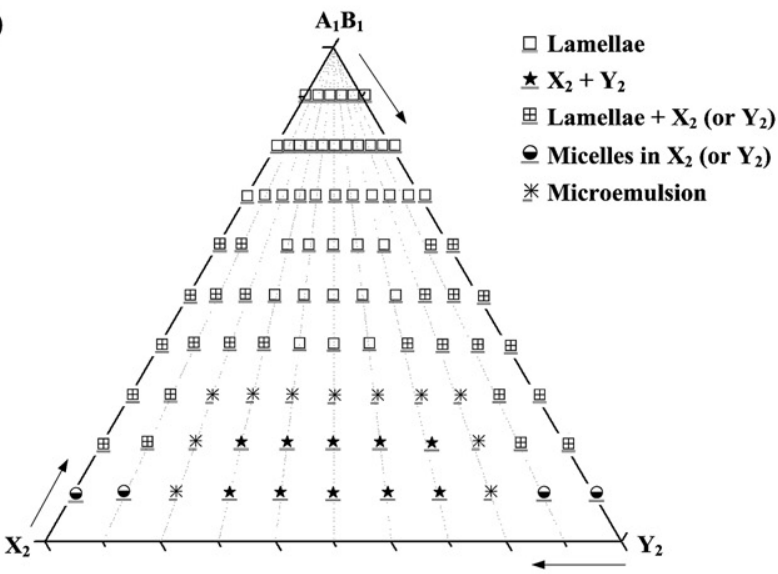

(c)

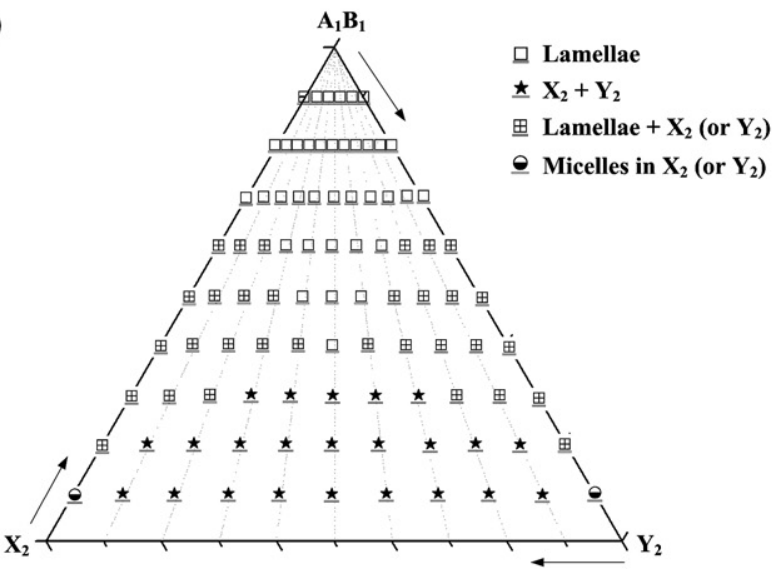

Fig. 2. Phase diagrams of symmetric $A_{1} B_{1}$ in the presence of A-selective solvent $\mathrm{X}_{2}$ and B-selective solvent $\mathrm{Y}_{2}$ for (a) $a_{\mathrm{XY}}=25$, (b) $a_{\mathrm{XY}}=30$, and (c) $a_{\mathrm{XY}}=40$. The other interaction parameters are set as $a_{\mathrm{AB}}=a_{\mathrm{AY}}=a_{\mathrm{BX}}=$ 100.54 , and $a_{\mathrm{AX}}=a_{\mathrm{BY}}=25$.

diagram in Fig. 2(b). This is not surprising since increasing the solvent immiscibility also enhances the preferentiality of $\mathrm{X}_{2}$ for $\mathrm{A}$ and $\mathrm{Y}_{2}$ for $\mathrm{B}$. Accordingly, as $a_{\mathrm{XY}}$ increases, more $\mathrm{X}_{2}$ and $\mathrm{Y}_{2}$ can be dissolved into the A-rich and B-rich domains, respectively. By varying the ratio of solvent amount $\bar{\phi}_{\mathrm{X}_{2}} / \bar{\phi}_{\mathrm{Y}_{2}}$, we obtain various phase transition behaviors with $\bar{\phi}_{\mathrm{C}}$. For example, the systems in which the amount of $\mathrm{X}_{2}$ is much more than that of $\mathrm{Y}_{2}$, undergo a sequence of $1 \mathrm{~L}$
$\left(\mathrm{A} / \mathrm{X}_{2}\right.$-rich and $\mathrm{B} / \mathrm{Y}_{2}$-rich $) \rightarrow 1 \mathrm{~L}\left(\mathrm{~A} / \mathrm{X}_{2}\right.$-rich and $\mathrm{B} / \mathrm{Y}_{2}$-rich $)+$ $1 \mathrm{D}\left(\right.$ solvent $\left.\mathrm{X}_{2}\right) \rightarrow$ micelles in excess solvent $\mathrm{X}_{2}$ as $\bar{\phi}_{\mathrm{C}}$ decreases. Similar phase transition behavior is also observed for the systems with $Y_{2}$ much more than $X_{2}$ except that the disordered phase becomes rich in solvent $\mathrm{Y}_{2}$. When the amounts of these two solvents $X_{2}$ and $Y_{2}$ are comparable, the solutions transform from $1 \mathrm{~L}\left(\mathrm{~A} / \mathrm{X}_{2}\right.$-rich and $\mathrm{B} / \mathrm{Y}_{2}$-rich $) \rightarrow$ microemulsion $\rightarrow 2$ disordered $\mathrm{X}_{2}$-rich and $\mathrm{Y}_{2}$-rich phases with decreasing $\bar{\phi}_{\mathrm{C}}$. Note that there exists a microemulsion region near the periphery of the $2 \mathrm{D}\left(\mathrm{X}_{2}+\mathrm{Y}_{2}\right)$ coexistence curve. As seen in Fig. 3(c) where we present the morphological pattern for the system with $\bar{\phi}_{\mathrm{C}}=0.2, \bar{\phi}_{\mathrm{X}_{2}} / \bar{\phi}_{\mathrm{Y}_{2}}=3 / 7$, and $a_{\mathrm{XY}}=30$, though $\mathrm{A}_{1} \mathrm{~B}_{1}$ mainly assembles along the interface between the $\mathrm{X}_{2}$-rich and $\mathrm{Y}_{2}$-rich segregated domains, but in fact the interface is not very obvious. If we further increase the immiscibility parameter between solvents $\mathrm{X}_{2}$ and $\mathrm{Y}_{2}$ to 40 (the corresponding phase diagram is presented in Fig. 2(c)), we find that the two-macrophase $\mathrm{X}_{2}$-rich and $\mathrm{Y}_{2}$-rich regimes significantly expands and even eats up the microemulsion phase region, as expected. For example, in Fig. 3(d) we present the corresponding morphological pattern for the same system as in Fig. 3(c) but $a_{\mathrm{XY}}$ increases to 40 . It is clear that both $\mathrm{X}_{2}$-rich and $\mathrm{Y}_{2}$-rich domains have sharp interfaces. Note that although the phase separation behavior of ternary mixtures of $\mathrm{A}_{1} \mathrm{~B}_{1}, \mathrm{X}_{2}$, and $\mathrm{Y}_{2}$ at higher immiscibility parameter $a_{\mathrm{XY}}$ is not examined here, it is reasonable to expect three-phase regions rich in each component, respectively, in the phase diagrams.

To examine the effects of adding two solvents $\mathrm{X}_{2}$ and $\mathrm{Y}_{2}$ on the phase behavior of asymmetric amphiphilic molecules, we choose $A_{1} B_{3}$ as a representative. In this case, pure $A_{1} B_{3}$ forms a stable $\mathrm{C}_{\mathrm{A}}^{\mathrm{HEX}}$. Fig. 4 presents a series of phase diagrams for $A_{1} B_{3}$ in the presence of two solvents $X_{2}$ and $Y_{2}$ at various values of the immiscibility parameter $a_{\mathrm{XY}}$. When only one solvent is added, the resulting phase transitions are straightforward, as have been discussed in the case of symmetric $A_{1} B_{1}$. In the $\mathrm{A}$-selective solvent $\mathrm{X}_{2}$ which is strongly immiscible with the majority $\mathrm{B}$ blocks, we observe a stable $\mathrm{C}_{\mathrm{A}}^{\mathrm{HEX}}$ phase at $\bar{\phi}_{\mathrm{C}} \geq 0.8$ and a macrophase separation into two phases rich in $\mathrm{A}_{1} \mathrm{~B}_{3}$ and $\mathrm{X}_{2}$, respectively, at $0.1 \leq \bar{\phi}_{\mathrm{C}} \leq 0.7$. In the $\mathrm{B}$-selective solvent $\mathrm{Y}_{2}$, because of the fact that the strongly immiscible A block is a minority component in the amphiphilic molecules, the addition of $\mathrm{Y}_{2}$ can steadily partition into the B-rich domains. As a result, a sequence of microphase transition of $\mathrm{C}_{\mathrm{A}}^{\mathrm{HEX}}\left(\bar{\phi}_{\mathrm{C}} \geq 0.8\right) \rightarrow$ A-formed long or short micelles $\left(\bar{\phi}_{\mathrm{C}}=0.7,0.6\right) \rightarrow$ A-formed spheres $\left(\mathrm{S}_{\mathrm{A}}\right)\left(\bar{\phi}_{\mathrm{C}} \leq 0.5\right)$ occurs. Note that this sequential microphase transition behavior induced by the addition of a selective solvent has been in a qualitatively good agreement with our previous SCMF results except the spherical ordering phase [30]. Recall that both experimental [29] and theoretical [30] studies have confirmed that the "normal" spheres (i.e., formed by the minority blocks) adopt a body-centered cubic (bcc) lattice while the "inverted" spheres (i.e., formed by the majority blocks) tend to pack from bcc to face-centered cubic (fcc) upon increasing the solvent selectivity and/or solvent amount. However, our DPD results demonstrate that the packing array of the spheres is strongly dependent on the size of the simulation box, which will be discussed later. 

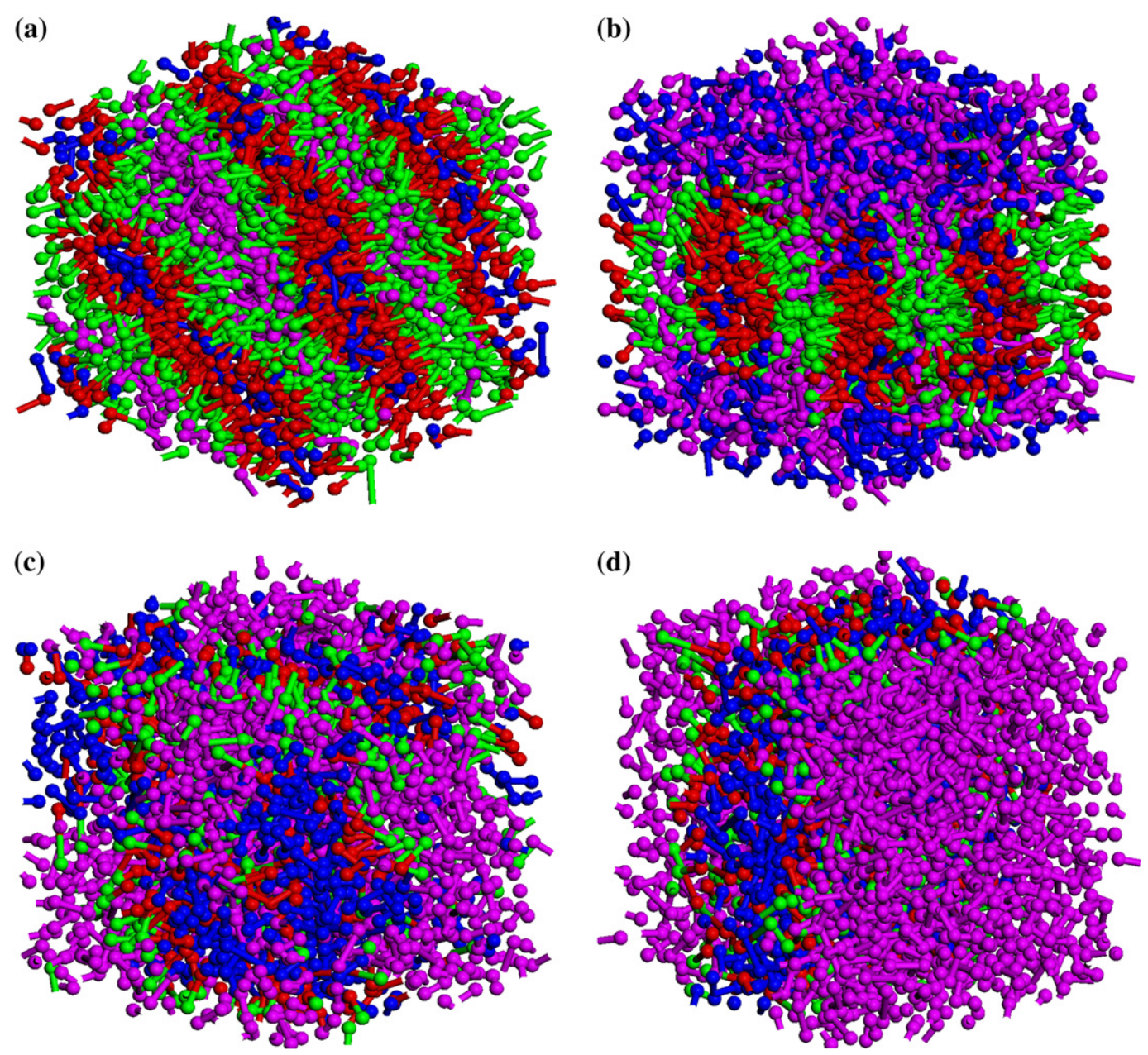

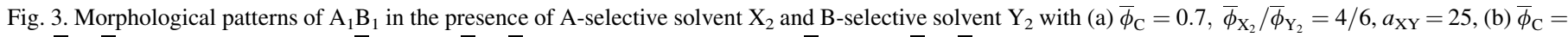

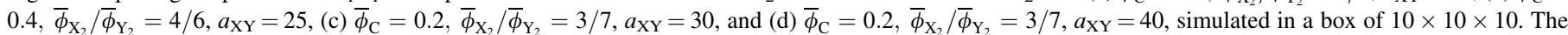

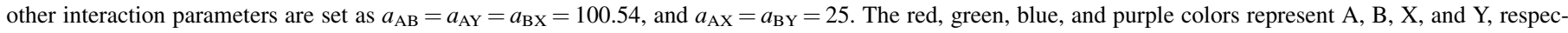
tively. (For interpretation of the references to color in this figure legend, the reader is referred to the web version of this article.)

When both solvents are added simultaneously, the effects of the immiscibility parameter between two solvents $a_{\mathrm{XY}}$ on the phase behavior of asymmetric $\mathrm{A}_{1} \mathrm{~B}_{3}$ (Fig. 4) are similar to that of symmetric $A_{1} B_{1}$ (Fig. 2). For example, when the solutions are concentrated $\left(\bar{\phi}_{\mathrm{C}} \geq 0.8\right)$ so that the amounts of $\mathrm{X}_{2}$ and $\mathrm{Y}_{2}$ which partition into A-rich and B-rich domains are relatively small, the original microstructure formed by pure $\mathrm{A}_{1} \mathrm{~B}_{3}$, i.e., $\mathrm{C}_{\mathrm{A}}^{\mathrm{HEX}}$, can be preserved regardless of the value of $a_{\mathrm{XY}}$ and $\bar{\phi}_{\mathrm{X}_{2}} / \bar{\phi}_{\mathrm{Y}_{2}}$. The typical $\mathrm{C}_{\mathrm{A}}^{\mathrm{HEX}}$ structural pattern is presented in Fig. $5(\mathrm{a})$, where $\bar{\phi}_{\mathrm{C}}=0.9, \bar{\phi}_{\mathrm{X}_{2}} / \bar{\phi}_{\mathrm{Y}_{2}}=5 / 5$, and $a_{\mathrm{XY}}=25$. For the solutions with $\bar{\phi}_{\mathrm{C}}$ in the range between 0.1 and 0.7 , the morphology type is greatly influenced by $a_{\mathrm{XY}}$ and $\bar{\phi}_{\mathrm{X}_{2}} / \bar{\phi}_{\mathrm{Y}_{2}}$. When $a_{\mathrm{XY}}=25$, due to the fact that these two solvents are completely miscible, the extra amount of solvents forms a miscible $X_{2} / Y_{2}$ disordered phase, as expected. That is, the systems undergo a macrophase separation into an $A_{1} B_{3}$-rich phase and a disordered $X_{2} / Y_{2}$ phase, as in the $A_{1} B_{1}$ systems. Although $A_{1} B_{3}$ forms a phase itself, the microstructure type varies with the ratio of solvent amount. When $\mathrm{X}_{2}$ is the major solvent, $\mathrm{A}_{1} \mathrm{~B}_{3}$ only aggregates as a macroscopic domain, as presented in Fig. 5(b), where $\bar{\phi}_{\mathrm{C}}=0.6, \bar{\phi}_{\mathrm{X}_{2}} / \bar{\phi}_{\mathrm{Y}_{2}}=$
$7 / 3$, and $a_{\mathrm{XY}}=25$. Nevertheless, when $\mathrm{Y}_{2}$ becomes the major solvent, $\mathrm{A}_{1} \mathrm{~B}_{3}$ forms micelles with $\mathrm{A}$ and $\mathrm{X}_{2}$ inside the cores and coexists with a disordered $\mathrm{X}_{2} / \mathrm{Y}_{2}$ miscible phase. In more detail, when the solutions are more concentrated, these A-formed micelles are cylindrical, and the typical pattern is shown in Fig. 5(c), where $\bar{\phi}_{\mathrm{C}}=0.6, \bar{\phi}_{\mathrm{X}_{2}} / \bar{\phi}_{\mathrm{Y}_{2}}=3 / 7$, and $a_{\mathrm{XY}}=25$. As $\bar{\phi}_{\mathrm{C}}$ decreases and/or the proportion of $\mathrm{Y}_{2}$ amount in the solvents increases, these A-formed cylinders become shorter and even transform into spheres, as in Fig. 5(d) where $\bar{\phi}_{\mathrm{C}}=0.2, \bar{\phi}_{\mathrm{X}_{2}} / \bar{\phi}_{\mathrm{Y}_{2}}=2 / 8$. This transition of cylinders into spheres is not surprising since increasing the ratio of $\mathrm{Y}_{2}$ amount in the two miscible solvents resembles a consequence of enhancing the solvent selectivity for B. As the solvent immiscibility parameter $a_{\mathrm{XY}}$ increases, similar to that observed in $\mathrm{A}_{1} \mathrm{~B}_{1}$, the miscible $\mathrm{X}_{2} / \mathrm{Y}_{2}$ disordered phase, which exists in the regime of $0.1 \leq \bar{\phi}_{\mathrm{C}} \leq 0.7$ at $a_{\mathrm{XY}}=25$, is no longer stable. It is interesting to find that the solutions with $\mathrm{Y}_{2}$ as the major solvent, which originally separate into $\mathrm{A}_{1} \mathrm{~B}_{3}$-rich and $\mathrm{X}_{2} / \mathrm{Y}_{2}$-rich phases at $a_{\mathrm{XY}}=25$, form spheres with minority $\mathrm{A}$ and $\mathrm{X}_{2}$ in the cores and majority $\mathrm{B}$ and $\mathrm{Y}_{2}$ in the matrix with increasing $a_{\mathrm{XY}}$. This is reasonable since increasing the solvent 
(a)

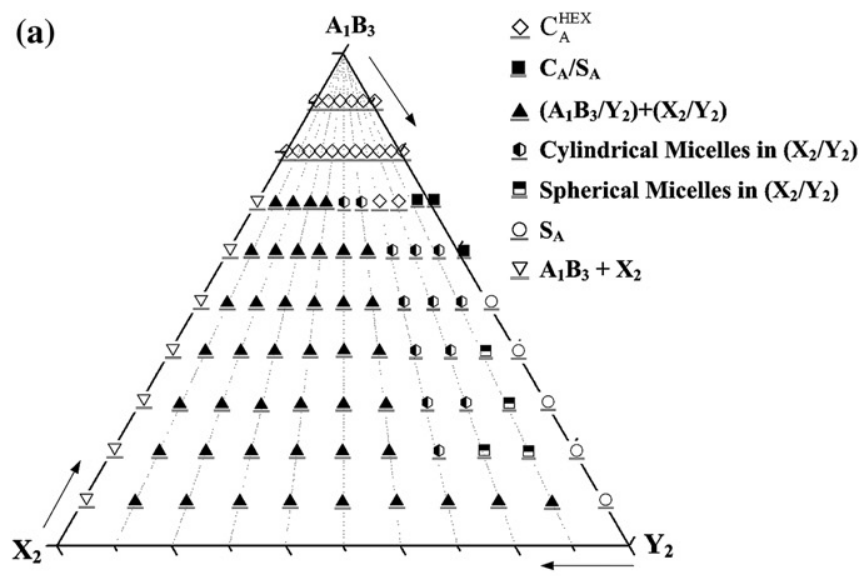

(b)

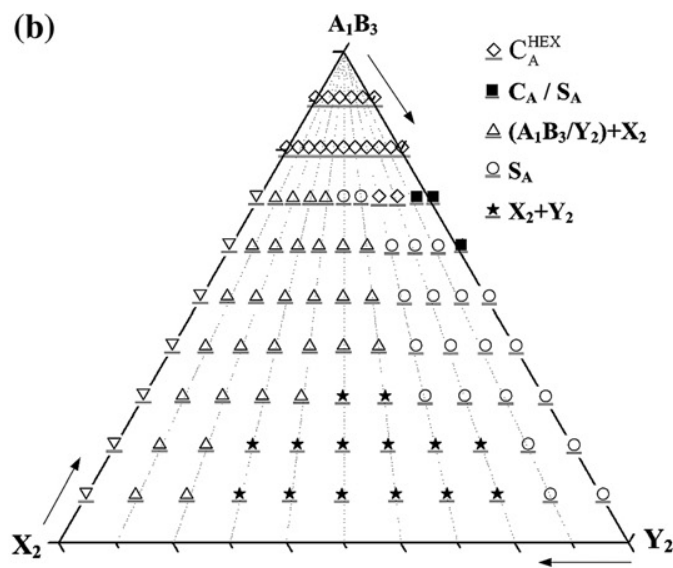

(c)

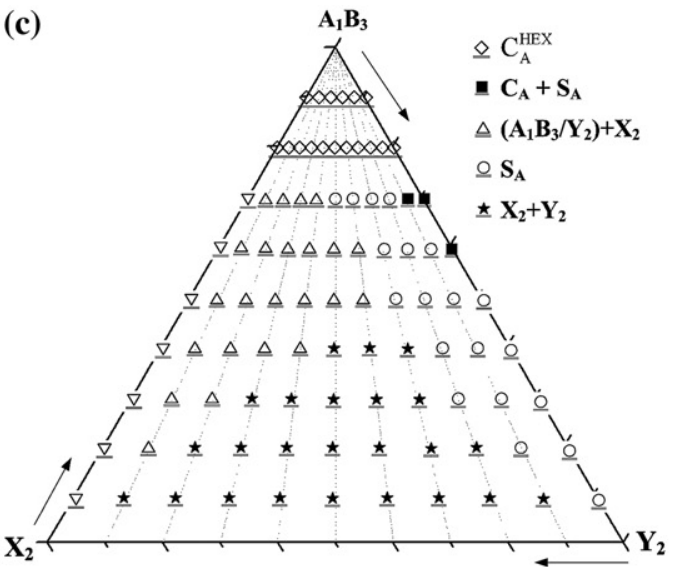

Fig. 4. Phase diagrams of asymmetric $A_{1} B_{3}$ in the presence of A-selective solvent $\mathrm{X}_{2}$ and B-selective solvent $\mathrm{Y}_{2}$ for (a) $a_{\mathrm{XY}}=25$, (b) $a_{\mathrm{XY}}=30$, and (c) $a_{\mathrm{XY}}=40$. The other interaction parameters are set as $a_{\mathrm{AB}}=a_{\mathrm{AY}}=a_{\mathrm{BX}}=$ 100.54 , and $a_{\mathrm{AX}}=a_{\mathrm{BY}}=25$.

immiscibility enhances the preferentiality of $\mathrm{X}_{2}$ for $\mathrm{A}$ and $\mathrm{Y}_{2}$ for $B$ and thus qualitatively increases the degree of molecular asymmetry for $A_{1} B_{3}$ when $Y_{2}$ is the major solvent. As a result, we observe a significantly enlarged microstructure region induced by increasing $a_{\mathrm{XY}}$ in the region when $\mathrm{Y}_{2}$ amount is more than $\mathrm{X}_{2}$.

Based on the fact that the effects of adding two strongly selective solvents on the resulting phase behavior of $A_{1} B_{1}$ and $A_{1} B_{3}$ are qualitatively consistent, we expect that similar results also hold true qualitatively in other amphiphilic molecules $\left(\mathrm{A}_{m} \mathrm{~B}_{n}\right)$ which initially form different ordered phases, such as G, PL, and S. As in the amphiphilic molecule solutions when only one solvent is added, in addition to the macrophase separation, a sequential transition of microstructures including $\mathrm{L}, \mathrm{G}, \mathrm{PL}, \mathrm{C}$, and $\mathrm{S}$ is possible by varying the immiscibility parameter between two solvents $a_{\mathrm{XY}}$, the ratio of solvent amount $\bar{\phi}_{\mathrm{X}_{2}} / \bar{\phi}_{\mathrm{Y}_{2}}$, and the volume fraction of amphiphilic molecules $\bar{\phi}_{\mathrm{C}}$. Though the addition of a second solvent cannot induce new morphology types, the presence of two solvents enriches the phase behavior further. When the added two solvents are miscible, a typical transition of 1 ordered phase $\rightarrow$ coexistence of $\mathrm{A}_{m} \mathrm{~B}_{n}$-rich and $\mathrm{X}_{2} / \mathrm{Y}_{2}$-rich phases occurs with decreasing $\bar{\phi}_{\mathrm{C}}$. Increasing the immiscibility between two solvents $a_{\mathrm{XY}}$ significantly enlarges the 1 ordered phase regime, in which a series of microphase transition can even be induced. This transition behavior is analogous to qualitatively varying the degree of molecular asymmetry for $\mathrm{A}_{m} \mathrm{~B}_{n}$. Furthermore, we infer that these results associated with varying the solvent immiscibility parameter are quite general for the amphiphilic molecules in the presence of two solvents, as they have also been observed in our previous phase behavior study of $\mathrm{AB}$ diblock copolymers in the presence of one neutral solvent and one slightly selective solvent [31].

The last issue to consider is whether the above mesophase behavior results are dependent on the finite size of the simulation box. In order to manifest this, we examine each microstructure in different sizes of the simulation box $L^{3}$ with $L \geq 10$. When the systems form the lamellar and hexagonally packed cylindrical phases, as long as the simulation box size is significantly larger than the radius of gyration of amphiphilic molecules, we observe that these ordered structures are no longer affected by the simulation box. However, when the amphiphilic molecule systems tend to form the spherical micelles, we find that the packing array of these spheres is strongly dependent on the size of the simulation box even though the box size is much larger than the radius of gyration of amphiphilic molecules. For example, in Fig. 6(a)-(c) we present the patterns for $\mathrm{A}_{1} \mathrm{~B}_{3}$ in the solvents $\mathrm{X}_{2}$ and $\mathrm{Y}_{2}$ with $\bar{\phi}_{\mathrm{C}}=$ $0.6, \bar{\phi}_{\mathrm{X}_{2}} / \bar{\phi}_{\mathrm{Y}_{2}}=2 / 8$, and $a_{\mathrm{XY}}=30$, simulated in a box of $10^{3}, 13^{3}$, and $16^{3}$, respectively. It is clear that the spherical micelles with the radius approximately equal to 2.6 grids are formed in the box of $10^{3}, 13^{3}$, and $16^{3}$, respectively, but they pack into an fcc, A15, and bcc lattice, respectively. With a further inspection of Fig. 6(a) the number of the effective spheres formed in the simulation box of $10 \times 10 \times 10$ is equal to 4 , which simply corresponds to the value of effective spheres in an fcc lattice. Due to the fact that the radius of the formed spheres is independent of the simulation box, the number of the effective spheres allowed to form in the box of $13 \times 13 \times 13$ and $16 \times 16 \times 16$ is thus expected to be equal to $4 \times(1.3)^{3} \cong 8$ and $4 \times(1.6)^{3} \cong 16$, respectively. Recall that the number of effective spheres in the A15 lattice and bcc lattice is 8 and 2, respectively. Therefore, it is not surprising that the spheres in the box of $13 \times 13 \times 13$ form an A15 lattice (Fig. 6(b)), and they pack into a bcc array in the box of $16 \times 16 \times 16$, which includes 8 unit cells (Fig. 6(c)) . 
(a)

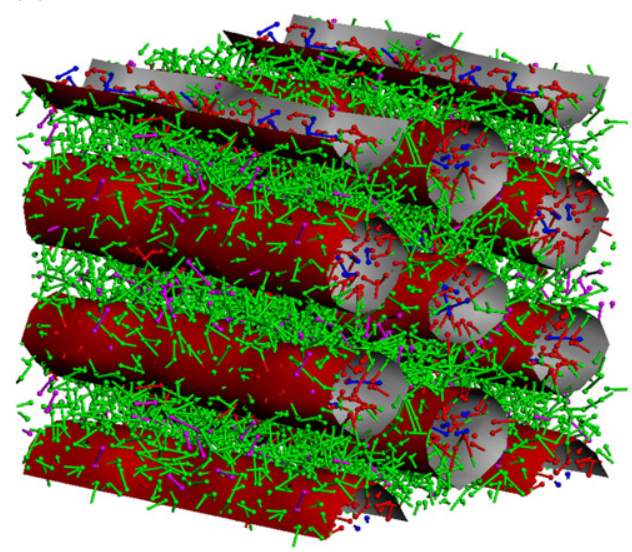

(c)

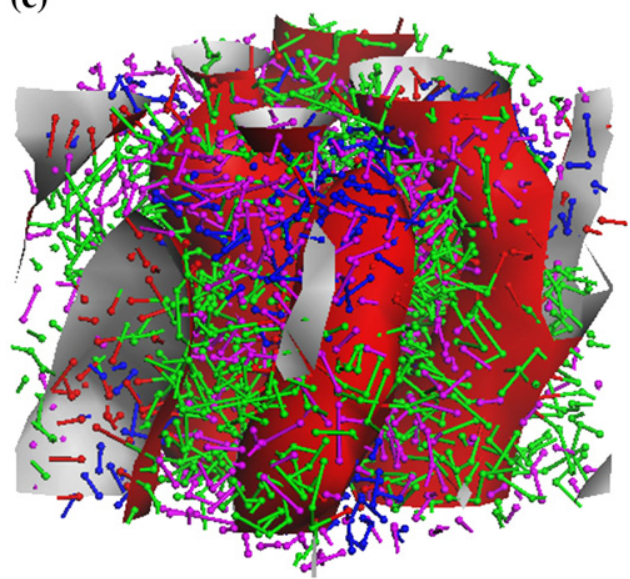

(b)

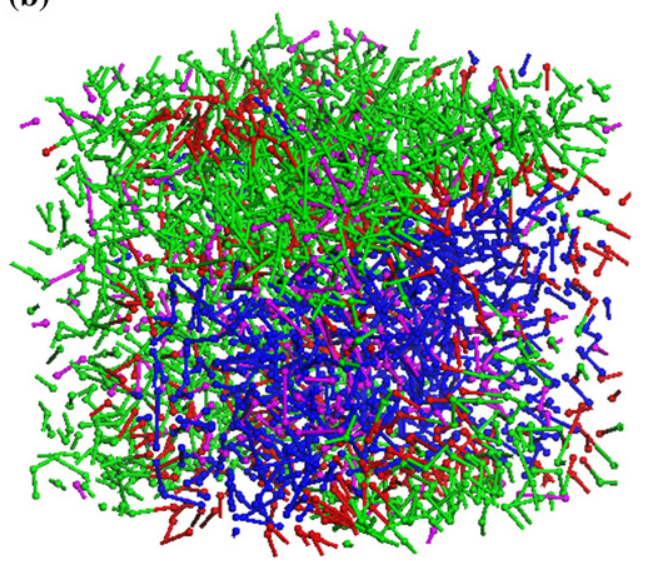

(d)

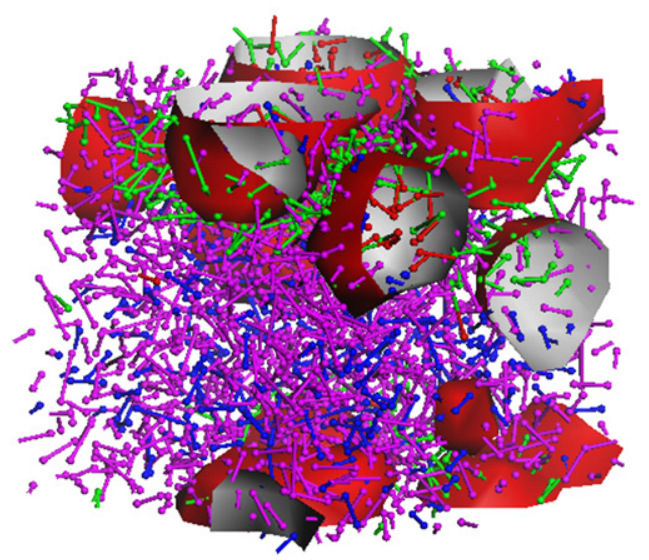

Fig. 5. Morphological patterns of $\mathrm{A}_{1} \mathrm{~B}_{3}$ in the presence of A-selective solvent $\mathrm{X}_{2}$ and B-selective solvent $\mathrm{Y}_{2}$ when $a_{\mathrm{XY}}=25$, and (a) $\bar{\phi}_{\mathrm{C}}=0.9, \bar{\phi}_{\mathrm{X}_{2}} / \bar{\phi}_{\mathrm{Y}_{2}}=5 / 5$, (b) $\bar{\phi}_{\mathrm{C}}=0.6, \bar{\phi}_{\mathrm{X}_{2}} / \bar{\phi}_{\mathrm{Y}_{2}}=7 / 3$, (c) $\bar{\phi}_{\mathrm{C}}=0.6, \bar{\phi}_{\mathrm{X}_{2}} / \bar{\phi}_{\mathrm{Y}_{2}}=3 / 7$, and (d) $\bar{\phi}_{\mathrm{C}}=0.2, \bar{\phi}_{\mathrm{X}_{2}} / \bar{\phi}_{\mathrm{Y}_{2}}=2 / 8$, simulated in a box of $10 \times 10 \times 10$. The other interaction parameters are set as $a_{\mathrm{AB}}=a_{\mathrm{AY}}=a_{\mathrm{BX}}=100.54$, and $a_{\mathrm{AX}}=a_{\mathrm{BY}}=25$. The red, green, blue, and purple colors represent $\mathrm{A}, \mathrm{B}, \mathrm{X}$, and Y, respectively. The red surface corresponds to the isosurface of A. (For interpretation of the references to color in this figure legend, the reader is referred to the web version of this article.)

The reason that these bcc, fcc, and A15 packing arrays are possible to occur for the same systems but in different sizes of the simulation box via DPD may be due to the fact that the free energy of these spherical packing lattices is quite close. Generally speaking, though DPD may not identify the most stable packing array of spheres due to the significant finite size effects, it reveals the possibility for the spheres packing into an A15 lattice. Indeed, in addition to fcc and bcc, A15 has been proposed as a quite possible state in the amphiphilic molecule systems [32].

In general, our current results reveal that the DPD simulation method has successfully captured the microphase separation behavior associated with lamellae and hexagonally packed cylinders and the macrophase separation behavior of an amphiphilic molecule in the presence of two solvents. Various transitions with decreasing $\bar{\phi}_{\mathrm{C}}$ occur by varying the interaction parameter $a_{\mathrm{XY}}$ and solvent ratio $\bar{\phi}_{\mathrm{X}_{2}} / \bar{\phi}_{\mathrm{Y}_{2}}$. However, we find that the packing order of the spherical micelles is greatly affected by the finite size of the simulation box. As such, it may become difficult to examine the stability of the spherical ordered phases via the DPD method.

\section{Summary}

We employ dissipative particle dynamics (DPD) to study the phase behavior of $\mathrm{A}_{m} \mathrm{~B}_{n}$ amphiphilic molecules in the presence of two solvents $\mathrm{X}_{2}$ and $\mathrm{Y}_{2}$, which are strongly selective for $\mathrm{A}$ and $\mathrm{B}$, respectively. As in the amphiphilic molecule solutions when only one solvent is added, in addition to the macrophase separation, the possible formed phases include $\mathrm{L}, \mathrm{G}$, PL, C, and S. Though the addition of a second solvent cannot induce new morphology types, the phase behavior is strongly affected by the immiscibility parameter between two solvents $a_{\mathrm{XY}}$, the ratio of solvent amount $\bar{\phi}_{\mathrm{X}_{2}} / \bar{\phi}_{\mathrm{Y}_{2}}$, and the volume fraction of amphiphilic molecules $\bar{\phi}_{\mathrm{C}}$. We observe that increasing $a_{\mathrm{XY}}$ not only drives a macrophase separation into $\mathrm{X}_{2}$-rich and $\mathrm{Y}_{2}$-rich phases for systems at lower values of $\bar{\phi}_{\mathrm{C}}$, but also enlarges the 1 ordered microphase region at higher $\bar{\phi}_{\mathrm{C}}$. It even induces a sequential microphase transition, which is analogous to qualitatively varying the degree of molecular asymmetry in the amphiphilic molecules. This is reasonable due to the fact that increasing the solvent immiscibility enhances the preferentiality of $\mathrm{X}_{2}$ for $\mathrm{A}$ and $\mathrm{Y}_{2}$ for $\mathrm{B}$. Though the 

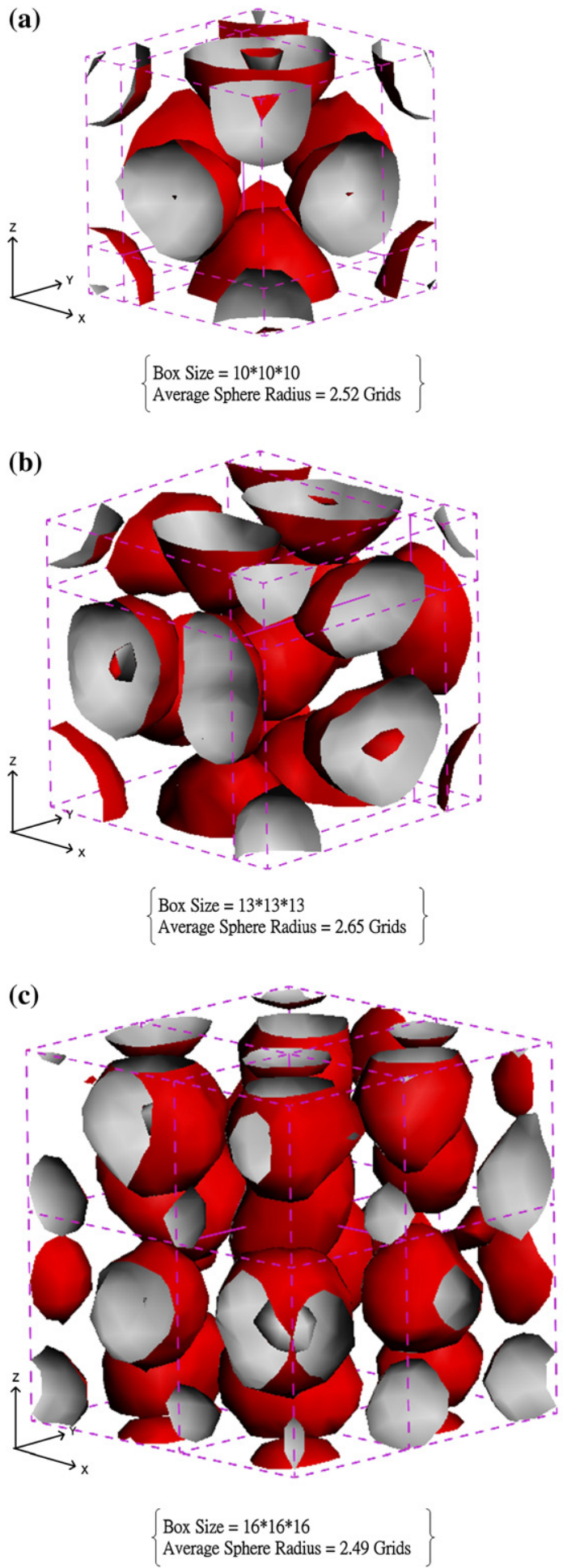
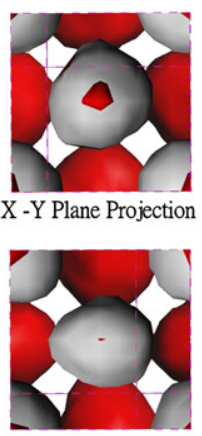

X - Z Plane Projection
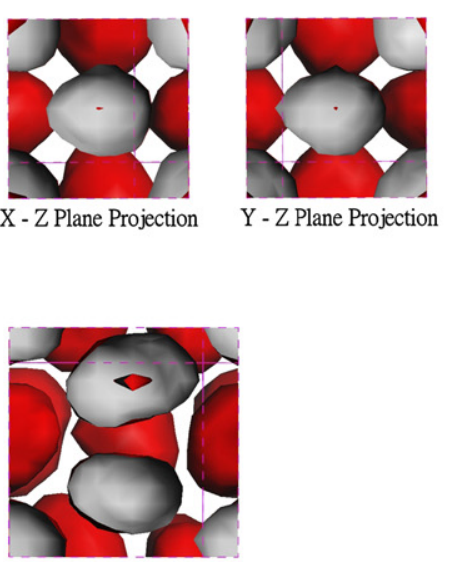

X -Y Plane Projection
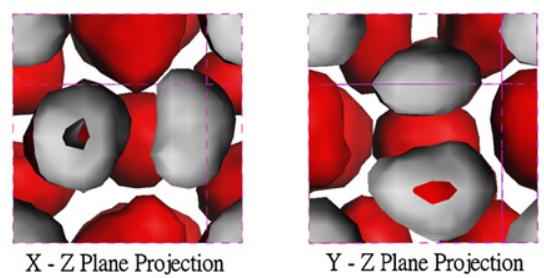

Y - Z Plane Projection

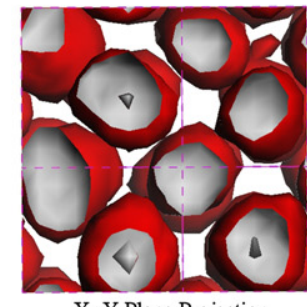

X -Y Plane Projection

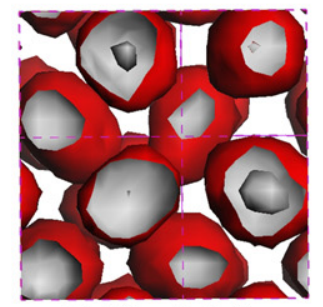

X - Z Plane Projection

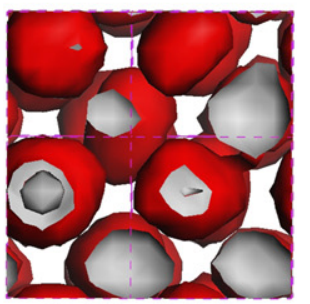

Y - Z Plane Projection

Fig. 6. Morphological patterns of $\mathrm{A}_{1} \mathrm{~B}_{3}$ in the presence of $\mathrm{X}_{2}$ and $\mathrm{Y}_{2}$ with $\bar{\phi}_{\mathrm{C}}=0.6, \bar{\phi}_{\mathrm{X}_{2}} / \bar{\phi}_{\mathrm{Y}_{2}}=2 / 8$, and $a_{\mathrm{XY}}=30$, simulated in a box of (a) $10 \times 10 \times 10$, (b) $13 \times 13 \times 13$, and (c) $16 \times 16 \times 16$. The other interaction parameters are set as $a_{\mathrm{AB}}=a_{\mathrm{AY}}=a_{\mathrm{BX}}=100.54$, and $a_{\mathrm{AX}}=a_{\mathrm{BY}}=25$. The red, green, blue, and purple colors represent A, B, X, and Y, respectively. The red surface corresponds to the isosurface of component A. (For interpretation of the references to color in this figure legend, the reader is referred to the web version of this article.)

packing order of the spherical micelles is greatly affected by the finite size of the simulation box, DPD reveals a possible order of A15. Indeed, in addition to fcc and bcc, A15 has been proposed as a quite possible state in the amphiphilic molecule systems.

\section{Acknowledgements}

This work was supported by the National Science Council of the Republic of China through grant NSC 94-2216-E-002027. 


\section{References}

[1] Mouritsen OG. Life - as a matter of fact: the emerging science of lipidomics series. New York: Springer; 2005.

[2] Garti N, Spernath A, Aserin A, Lutz R. Soft Matter 2005;1:206.

[3] Hamley IW, editor. Developments in block copolymer science and technology. New Jersey: Wiley; 2004.

[4] Hadjichristidis N, Pispas S, Floudas G. Block copolymers: synthetic strategies, physical properties, and applications. New Jersey: Wiley; 2003.

[5] Lodge TP. Macromol Chem Phys 2003;204:265.

[6] Alexandridis P, Lindman B, editors. Amphiphilic block copolymers: self-assembly and applications. New York: Elsevier Science; 2000.

[7] Robinson BH, editor. Self-assembly. Amsterdam: IOS Press; 2003.

[8] Larson RG. J Phys II France 1996;6:1441.

[9] Mackie AD, Kaan O, Panagiotopoulos AZ. J Chem Phys 1996;104:3718.

[10] Talsania SK, Rodriguez-Guadarrama LA, Mohanty KK, Rajagopalan R. Langmuir 1998;14:2684.

[11] Kim SY, Panagiotopoulos AZ, Floriano MA. Mol Phys 2002;100:2213.

[12] Rekvig L, Kranenburg M, Vreede J, Hafskjold B, Smit B. Langmuir 2003; 19:8195.

[13] Schulz SG, Kuhn H, Schmid G, Mund C, Venzmer J. Colloid Polym Sci 2004;283:284.

[14] Hoogerbrugge P, Koelman J. Europhys Lett 1992;19:155.
[15] Groot RD, Warren PB. J Chem Phys 1997;107:4423.

[16] Groot RD, Madden TJ. J Chem Phys 1998;108:8713.

[17] Yamamoto S, Maruyama Y, Hyodo S. J Chem Phys 2002;116:5842.

[18] Ryjkina E, Kuhn H, Rehage H, Muller F, Peggau J. Angew Chem Int Ed 2002;41:983.

[19] Groot RD. Lect Notes Phys 2004;640:5.

[20] Kuo MY, Yang HC, Hua CY, Chen CL, Mao SZ, Deng F, et al. Chem Phys Chem 2004;5:575.

[21] Qian HJ, Lu ZY, Chen LJ, Li ZS, Sun CC. Macromolecules 2005;38: 1395.

[22] Cao X, Xu G, Li Y, Zhang Z. J Phys Chem A 2005;109:10418.

[23] Matsen MW, Bates FS. Macromolecules 1996;29:1091.

[24] Won YY, Davis HT, Bates FS, Agmalian M, Wignall GD. J Phys Chem B 2000;104:7134.

[25] Jakobs B, Sottmann T, Strey R, Allgaier J, Willner L, Richter D. Langmuir 1999;15:6707.

[26] Espanol P, Warren PB. Europhys Lett 1995;30:191.

[27] Allen MP, Tildesley DJ. Computer simulation of liquids. Oxford: Clarendon; 1987.

[28] Janert PK, Schick M. Macromolecules 1998;31:1109.

[29] Lodge TP, Pudil B, Hanley KJ. Macromolecules 2002;35:4707.

[30] Huang CI, Hsueh HY. Polymer 2006;47:6843.

[31] Huang CI, Hsu YC. Phys Rev E 2006;74:051802.

[32] Ziherl P, Kamien RD. J Phys Chem B 2001;105:10147. 